\title{
An Efficient Algorithm for Instantaneous Frequency Estimation of Nonstationary Multicomponent Signals in Low SNR
}

\author{
Jonatan Lerga, ${ }^{1}$ Victor Sucic (EURASIP Member), ${ }^{1}$ and Boualem Boashash ${ }^{2,3}$ \\ ${ }^{1}$ Faculty of Engineering, University of Rijeka, Vukovarska 58, 51000 Rijeka, Croatia \\ ${ }^{2}$ College of Engineering, Qatar University, P.O. Box 2713, Doha, Qatar \\ ${ }^{3}$ UQ Centre for Clinical Research, The University of Queensland, Brisbane QLD 4072, Australia
}

Correspondence should be addressed to Victor Sucic, vsucic@riteh.hr

Received 14 July 2010; Revised 10 November 2010; Accepted 11 January 2011

Academic Editor: Antonio Napolitano

Copyright (C) 2011 Jonatan Lerga et al. This is an open access article distributed under the Creative Commons Attribution License, which permits unrestricted use, distribution, and reproduction in any medium, provided the original work is properly cited.

\begin{abstract}
A method for components instantaneous frequency (IF) estimation of multicomponent signals in low signal-to-noise ratio (SNR) is proposed. The method combines a new proposed modification of a blind source separation (BSS) algorithm for components separation, with the improved adaptive IF estimation procedure based on the modified sliding pairwise intersection of confidence intervals (ICI) rule. The obtained results are compared to the multicomponent signal ICI-based IF estimation method for various window types and SNRs, showing the estimation accuracy improvement in terms of the mean squared error (MSE) by up to $23 \%$. Furthermore, the highest improvement is achieved for low SNRs values, when many of the existing methods fail.
\end{abstract}

\section{Signal Model and Problem Formulation}

Many signals in practice, such as those found in speech processing, biomedical applications, seismology, machine condition monitoring, radar, sonar, telecommunication, and many other applications are nonstationary [1]. Those signals can be categorized as either monocomponent or multicomponent signals, where the monocomponent signal, unlike the multicomponent one, is characterized in the timefrequency domain by a single "ridge" corresponding to an elongated region of energy concentration [1].

For a real signal $s(t)$, its analytic equivalent $z(t)$ is defined as

$$
z(t)=s(t)+j \mathscr{H}\{s(t)\}=a(t) e^{j \phi(t)},
$$

where $\mathscr{H}\{s(t)\}$ is the Hilbert transformation of $s(t), a(t)$ is the signal instantaneous amplitude, and $\phi(t)$ is the signal instantaneous phase.

The instantaneous frequency (IF) describes the variations of the signal frequency contents with time; in the case of a frequency-modulated (FM) signal, the IF represents the FM modulation law and is often referred to as simply the IF law $[2,3]$. The IF of the monocomponent signal $z(t)$ is the first derivative of its instantaneous phase, that is, $\omega(t)=\phi^{\prime}(t)[1]$. Furthermore, the crest of the "ridge" is often used to estimate the IF of the signal $z(t)$ as [1]

$$
\hat{\omega}(t)=\arg \left[\max _{f} \operatorname{TFD}_{z}(t, f)\right],
$$

where $\operatorname{TFD}_{z}(t, f)$ is the signal $z(t)$ time-frequency distribution [1].

On the other hand, the analytical multicomponent signal $x(t)$ can be modeled as a sum of two or more monocomponent signals (each with its own IF $\omega_{m}(t)$ )

$$
x(t)=\sum_{m=1}^{M} z_{m}(t)=\sum_{m=1}^{M} a_{m}(t) e^{j \phi_{m}(t)},
$$

where $M$ is the number of signal components, $a_{m}(t)$ is the $m$ th component instantaneous amplitude, and $\phi_{m}(t)$ is its instantaneous phase.

When calculating the Hilbert transform of the signal $s(t)$ in (1), the conditions of Bedrosian's theorem need to be satisfied, that is, $a(t)$ has to be a low frequency function with the spectrum which does not overlap with the $e^{j \phi(t)}$ spectrum [2-5]. 
To obtain the multicomponent signal IF, a component separation procedure should precede the IF estimation from the extracted signal components [1]. However, when dealing with multicomponent signals, their TFDs often contain the cross-terms which significantly disturb signal timefrequency representation, hence making the components separation procedure more difficult. Thus the proper TFD selection plays a crucial role in signal components extraction efficiency. Various reduced interference distributions (RIDs) have been proposed in order to have a high resolution timefrequency signal representation, such as modified B distribution (MBD) [6] and the RID based on the Bessel kernel [7], both used in this paper. A measure for time-frequency resolution and component separation was proposed in [8].

Methods for signal components extraction from a mixture containing two or more statistically independent signals are often termed as blind source separation (BSS) methods, where the term "blind" indicates that neither the structure of the mixtures nor the source signals are known in advance [1]. So, the main problem of BSS is obtaining the original waveforms of the sources when only their mixture is available [9]. Due to its broad range of potential applications, BSS has attracted a great deal of attention, resulting in numerous BSS techniques which can be classified as the time domain methods (e.g., $[10,11])$, the frequency domain methods (e.g., [12, 13]), adaptive (recursive) methods (e.g., [14]), and nonrecursive methods (e.g., [15]).

Once the components are extracted from the signal, their IF laws (which describe the signal frequency modulation (FM) variation with time [1]) can be obtained using some of the existing IF estimation methods. One of the popular IF estimation methods is the iterative algorithm [16] based on the spectrogram calculated from the signal analytic associate in the algorithm's first iteration, followed by the IF and the instantaneous phase estimation. The obtained IF is then used for a new calculation of the spectrogram which is further used for signal demodulation. In the next iteration, the matched spectrogram of the demodulated signal is calculated, followed by a new IF and phase estimation. The procedure is iteratively repeated until the IF estimate convergence is reached (based on the threshold applied to the difference between consecutive iterations) [16].

The IF estimation methods for noisy signals can be divided into two categories comprising the case of multiplicative noise and the case of additive noise. For a signal in multiplicative noise or a signal with the timevarying amplitude, the use of the Wigner-Ville spectrum or the polynomial Wigner-Ville distribution was proposed in $[17,18]$.

For polynomial FM signals in additive noise and high signal-to-noise ratio (SNR), the polynomial Wigner-Ville distribution-based IF estimation method was suggested [19] while for the low SNR an iterative procedure based on the cross-polynomial Wigner-Ville distribution was proposed [20]. The signal polynomial phase, and its IF as the derivative of the obtained phase polynomial, can be also estimated using the higher-order ambiguity functions [21]. The IF estimation accuracy can be improved using the adaptive windows and the $S$-transform (which combines the short-time
Fourier analysis and the wavelet analysis) [22] or the directionally smoothed pseudo-Wigner-Ville distribution bank [23]. The IF estimation method based on the maxima of time-frequency distributions adapted using the intersection of confidence intervals (ICI) rule or its modifications, used in the varying data-driven window width selection, was shown to outperform the IF obtained from the maxima of the TFD calculated using the best fixed-size window width [24-26]. This paper presents a modification of the sliding pairwise ICI rule-based method for signal component IF estimation combined with the modified BSS method for components separation and extraction. Unlike the ICI rule based method which was used only for monocomponent signals, this new proposed method based on the improved ICI rule is extended and applied to multicomponent signals IF estimation, resulting into increased estimation accuracy for each component present in the signal.

A simplified flowchart of the new multicomponent IF estimation method is shown in Figure 1. As it can be seen, the components IF estimation using the proposed method is preceded by the modified component extraction procedure described in Section 2.3.

The paper is organized as follows. Section 2 gives an introduction to the problem of proper TFD selection, followed by the modified algorithm for components separation and extraction for multicomponent signals in additive noise. Section 3 defines the improved sliding pairwise ICI-based IF estimation method from a set of the signal TFDs calculated for various fixed window widths. Section 4 presents the results of the multicomponent signal IF estimation using the proposed method, and then compares them with the results obtained with the ICI-based method. The conclusion is given in Section 5.

\section{Components Extraction Procedure}

2.1. TFD Selection. When dealing with multicomponent signals, the choice of the TFD plays a crucial role due to the presence of the unwanted cross-terms which disturb the signal representation in the $(t, f)$ domain. The best-known TFD of a monocomponent linear FM analytic signal $z(t)$ is the Wigner-Ville distribution (WVD), which may be defined as [1]

$$
W_{z}(t, f)=\int_{-\infty}^{+\infty} z\left(t+\frac{\tau}{2}\right) \cdot z^{*}\left(t-\frac{\tau}{2}\right) \cdot e^{-j 2 \pi f \tau} d \tau .
$$

The main disadvantage of the WVD of multicomponent signals or monocomponent signals with nonlinear IF is the presence of interferences and loss of frequency resolution [1], as illustrated in Figure 2. To reduce the cross-terms in the WVD, the signal instantaneous autocorrelation function $z(t+\tau / 2) \cdot z^{*}(t-\tau / 2)$ can be windowed in the lag $\tau$ direction before taking its Fourier transform

$$
P W_{z}(t, f)=\int_{-\infty}^{+\infty} h(\tau) \cdot z\left(t+\frac{\tau}{2}\right) \cdot z^{*}\left(t-\frac{\tau}{2}\right) \cdot e^{-j 2 \pi f \tau} d \tau,
$$

resulting in the pseudo-WVD $P W_{z}(t, f)$ also called DopplerIndependent TFD [1, pages 213-214]. 


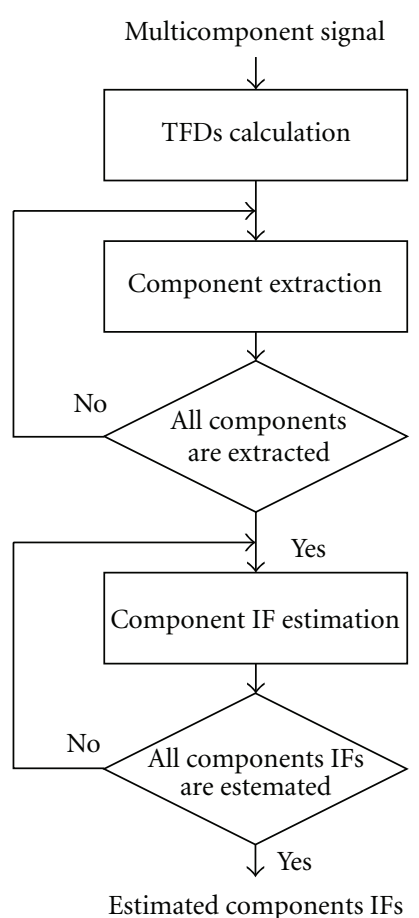

Figure 1: A simplified flowchart of the new IF estimation algorithm.

Narrowing the frequency smoothing window $h(\tau)$ of the pseudo WVD in order to better localize the signal in time results in higher TFD time resolution, and consequently lower frequency resolution [1]. Similarly, a narrow window in the frequency domain results in high frequency resolution, but simultaneously the time resolution gets disturbed [ 1 , page 215].

To have independently adjusted time and frequency smoothing of the WVD, the smoothed pseudo WVD was introduced [1]

$$
\begin{array}{r}
\operatorname{SPW}_{z}(t, f)=\int_{-\infty}^{+\infty} h(\tau) \int_{-\infty}^{+\infty} g(s-t) \cdot z\left(s+\frac{\tau}{2}\right) \\
\cdot z^{*}\left(s-\frac{\tau}{2}\right) d s \cdot e^{-j 2 \pi f \tau} d \tau,
\end{array}
$$

where $g(t)$ is the time smoothing window.

The efficiency of the IF estimation method presented in this paper is affected by the TFD selection, hence a reduced interference, high resolution TFD should be used. There are numerous TFDs having such characteristics, some of which are defined in [27-29]. One RID shown to be superior to other fixed-kernel TFDs in terms of cross-terms reduction and resolution enhancement, is the MBD defined as [6]

$$
\begin{array}{r}
\operatorname{MBD}_{z}(t, f)=\iint_{-\infty}^{+\infty} \frac{\cosh ^{-2 \beta}(t-u)}{\int_{-\infty}^{+\infty} \cosh ^{-2 \beta} \xi d \xi} \cdot z\left(u+\frac{\tau}{2}\right) \\
\cdot z^{*}\left(u-\frac{\tau}{2}\right) \cdot e^{-j 2 \pi f \tau} d u d \tau,
\end{array}
$$

where the parameter $\beta$, $(0<\beta \leq 1)$, controls the distribution resolution and cross-term elimination $[6,30]$. Generally, there is a compromise between those two TFD features, with the MBD shown to outperform many popular distributions $[6,8]$. Furthermore, the MBD was also proven to be a suitable TFD for robust IF estimation [6].

In this paper, the results obtained using the $\mathrm{MBD}$ are compared to those obtained by another RID with the kernel filter based on the Bessel function of the first kind (RIDB) [7]. This choice of the RID was motivated by its good performances in terms of time and frequency resolution preservation due to the independent windowing in the $\tau$ and $\nu$ domains, as well as its efficient cross-terms suppression [7]. The RIDB is defined as [7]

$$
\operatorname{RIDB}_{z}(t, f)=\int_{-\infty}^{+\infty} h(\tau) \cdot R_{z}(t, \tau) \cdot e^{-j 2 \pi f \tau} d \tau,
$$

where

$$
\begin{gathered}
R_{z}(t, \tau)=\int_{t-|\tau|}^{t+|\tau|} \frac{2 g(v)}{\pi|\tau|} \sqrt{1-\left(\frac{v-t}{\tau}\right)^{2}} \cdot z\left(v+\frac{\tau}{2}\right) \\
\cdot z^{*}\left(v-\frac{\tau}{2}\right) d v .
\end{gathered}
$$

This distribution has been tested on real-life signals, such as heart sound signal and Doppler blood flow signal, and proven to be superior over some other TFDs in suppressing the cross-terms, while the autoterms were kept with high resolution $[7,31,32]$.

2.2. Algorithm for Signal Components Extraction. The signal components separation and extraction can be done using the two algorithms given in [33], classified by their authors as the BSS algorithms even though they are different from standard BSS formulation, being ad hoc approaches. The first of those algorithms is applicable to multicomponent signals with intersecting components (assuming that all components have same time supports), while the second one is applicable to multicomponent signals with components which do not intersect and may have different time supports. The modifications proposed in this paper can be applied to both of these algorithms. However, in many practical situations that we have dealt with, components belonging to the same signal source do not generally intersect (e.g., newborn EEG seizure signal analysis [1]), so we have chosen to apply our modifications to the second algorithm only. Furthermore, the chosen algorithm, unlike some other algorithms for estimation of multicomponent signals in noise (e.g., [3436]) is not limited to the polynomial phase signals and can also be used in estimation of other nonlinear phase signals, as most real-life signals are (e.g., the echolocation sound emitted by a bat, used in this paper). The components are extracted one by one, until the remaining energy of the TFD becomes sufficiently small [37].

The algorithm consists of three major stages. In the first stage, a cross-terms free TFD, or the one with the cross-terms being suppressed as much as possible, is calculated. In the second stage of the algorithm, the components are extracted 


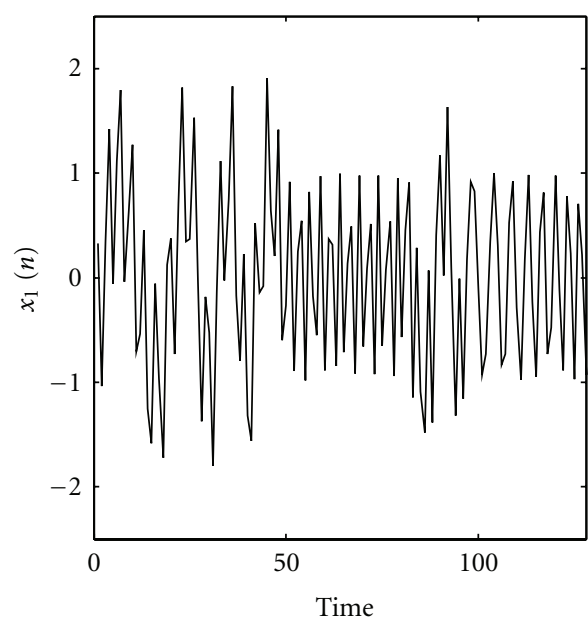

(a)

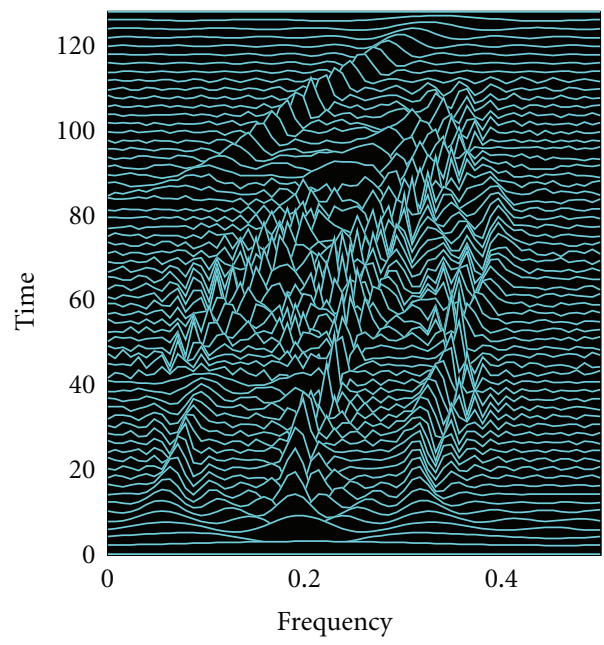

(c)

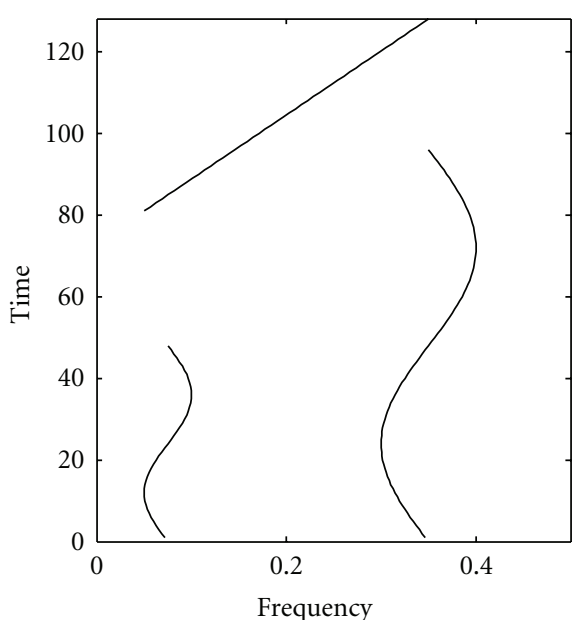

(b)

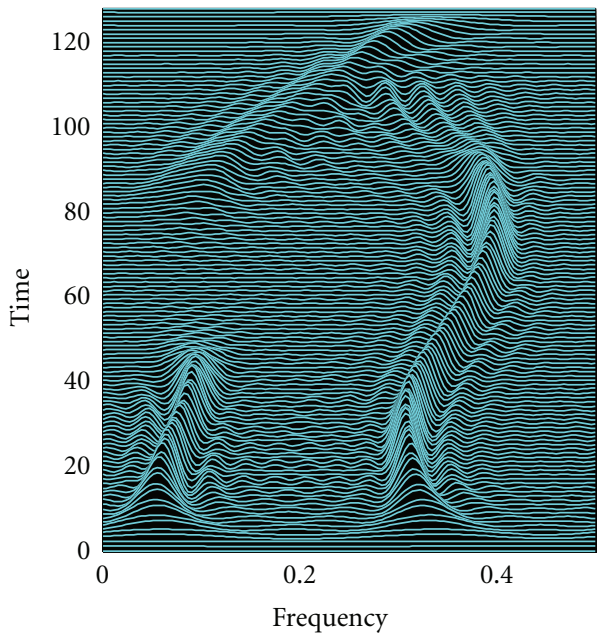

(d)

FIgURE 2: Example of a multicomponent signal and its representations in the $(t, f)$ domain. (a) Signal in the time domain. (b) Signal components IFs. (c) WVD of the signal. (d) Signal MBD with the time and lag window length of N/4 + 1 .

using the peaks of the TFD. The highest peak at $\left(t_{0}, f_{0}\right)$ in the time-frequency domain is extracted first, and then it is set to zero (in order to avoid it being selected again) along with the frequency range around it $\left(t_{0}, f\right)$ (the size of which is $2 \Delta f$, such that $\left.f \in\left[f_{0}-\Delta f, f_{0}+\Delta f\right]\right)$. Then the next highest peak $\left(t_{0}^{\prime}, f_{0}^{\prime}\right)$ in the vicinity of the previous one is selected. That is, $\left(t_{0}^{\prime}, f_{0}^{\prime}\right)$ is the maximum in the $(t, f)$ domain where $t \in\left[t_{0}-1, t_{0}+1\right]$ and $f \in\left[f_{0}-F / 2, f_{0}+F / 2\right]$, where $F$ is the chosen frequency window width. Next, $\left(t_{0}^{\prime}, f_{0}^{\prime}\right)$ is set to be $\left(t_{0}, f_{0}\right)$, and the procedure is repeated until the boundaries of the TFD are reached or the TFD value at $\left(t_{0}^{\prime}, f_{0}^{\prime}\right)$ is smaller than the preset threshold value $\epsilon_{c}$ defined as the fraction of the TFD value at the first $\left(t_{0}, f_{0}\right)$ point. Such extracted TFD's peaks constitute one signal component. The next component is found in the same way using the abovedescribed procedure. The algorithm stops once the energy remaining in the TFD is smaller than the threshold value $\epsilon_{d}$ defined as a fraction of the signal total energy.
The second stage of the algorithm often produces a number of components that is larger than the actual number of components present in the analyzed multicomponent signal. In order to fix this, a classification procedure was proposed as the third and final algorithm stage. This component classification procedure groups the components from the second stage of the algorithm based on the minimum distance between any pair of components. If two components belong to the same actual component, their distance is going to be smaller than the distance between the considered component and any other component, and they get combined into a single component [33].

\subsection{Modification of the Algorithm for Components Extraction.} In order to avoid the components classification procedure of the algorithm in [33], in this section, we present a modification of the components extraction algorithm. 


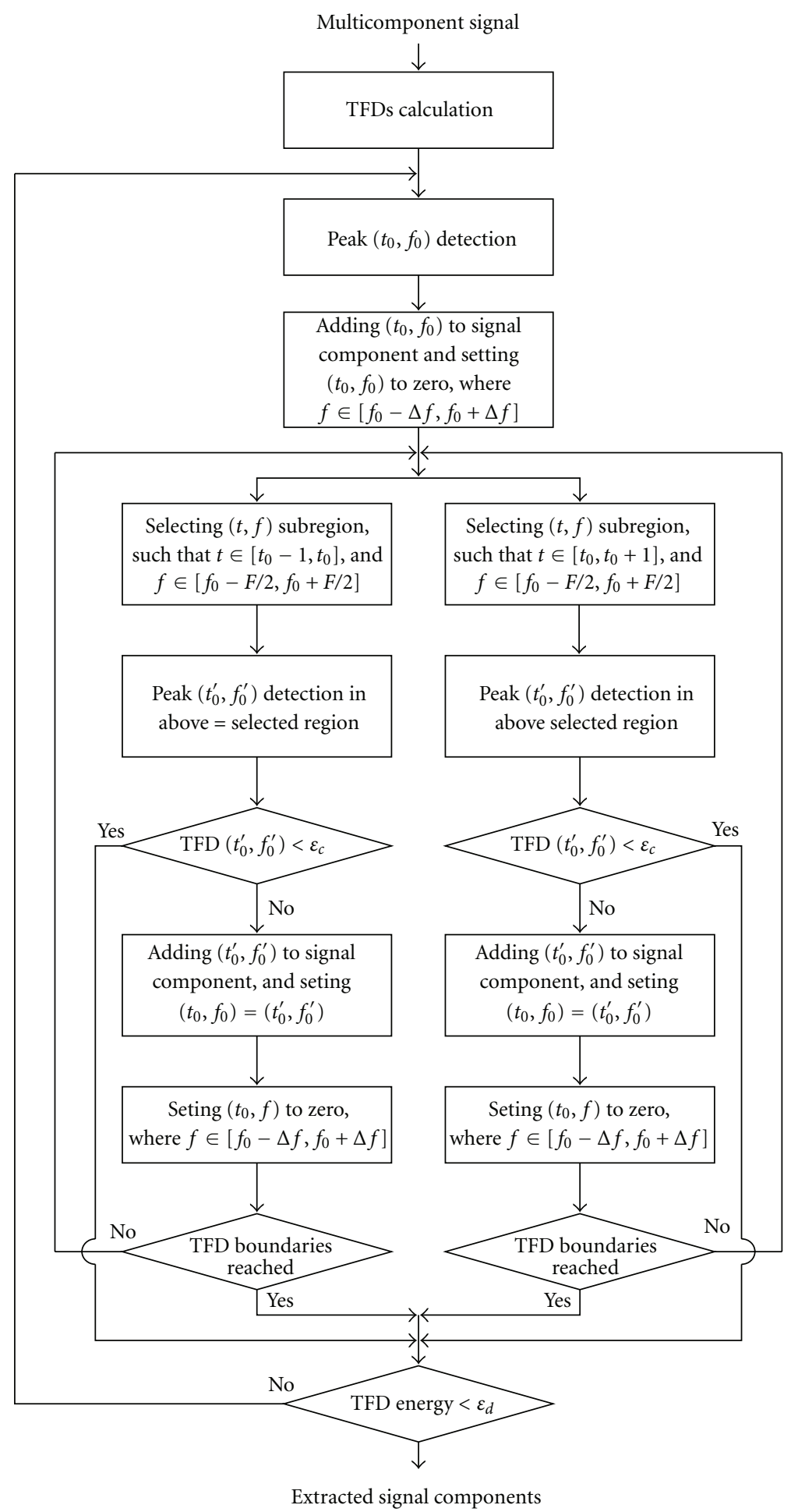

FIGURE 3: A detailed flowchart of the modified components extraction algorithm.

The modified algorithm (the flowchart of which is shown in Figure 3) for components separation and extraction starts with the signal RID calculation; the MBD and the RIDB are used in this paper.

As in the original method, the modified algorithm starts with the detection of the highest peak $\left(t_{0}, f_{0}\right)$ in the $(t, f)$ domain, followed by setting $\left(t_{0}, f\right)$ to zero, where $f \in\left[f_{0}-\right.$ $\left.\Delta f, f_{0}+\Delta f\right]$. Then, the $\left(t_{0}, f_{0}\right)$ vicinity is divided in two $(t, f)$ subregions such that $f \in\left[f_{0}-F / 2, f_{0}+F / 2\right]$, where $t \in$ $\left[t_{0}-1, t_{0}\right]$ for the first subregion and $t \in\left[t_{0}, t_{0}+1\right]$ for the second one. Thus, the two values for $\left(t_{0}^{\prime}, f_{0}^{\prime}\right)$ are obtained as the maximum of each of the two subregions. 


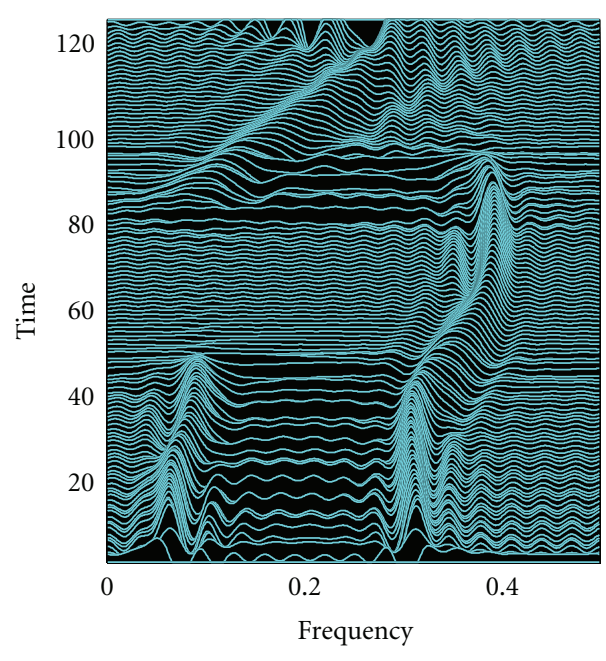

(a)

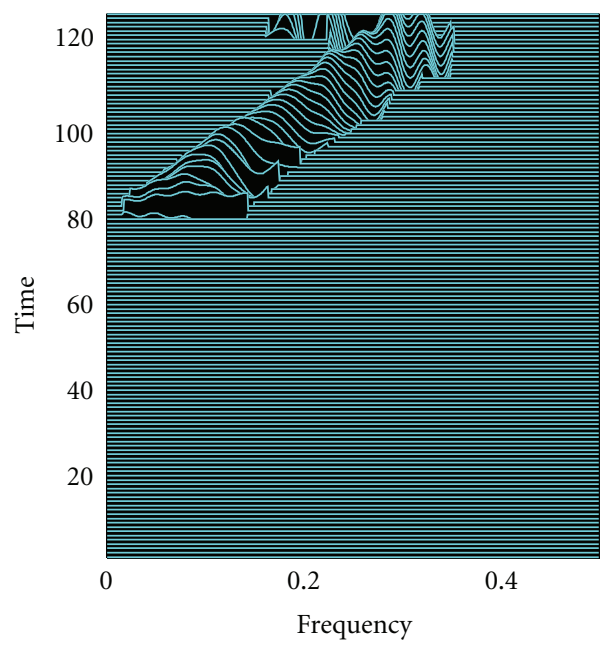

(c)

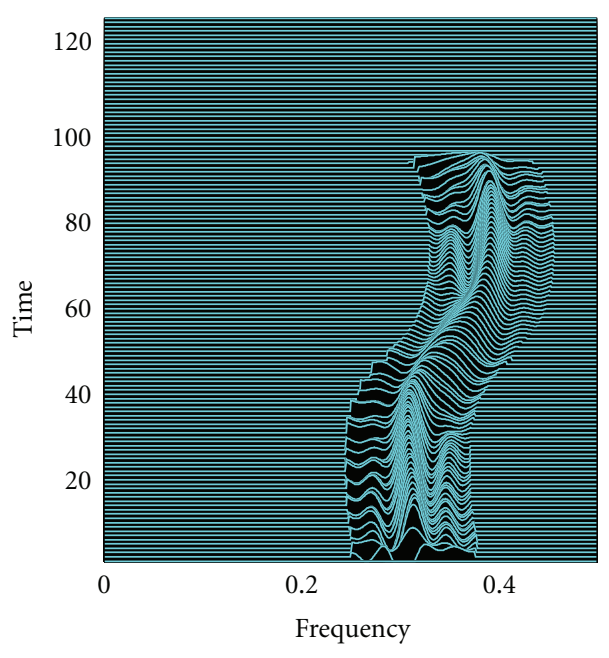

(b)

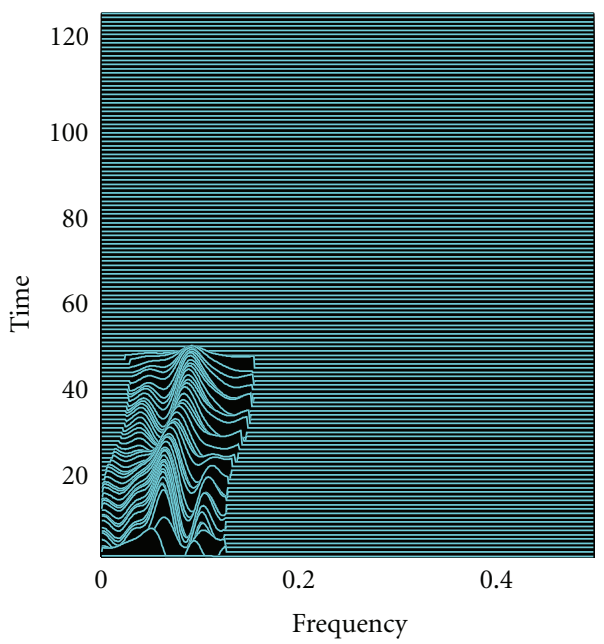

(d)

Figure 4: Example of components separation and extraction procedure using the algorithm described in Section $2(N=128$, the number of frequency bins $N_{f}=4 N, \Delta f=F / 2=N_{f} / 4, \epsilon_{c}=0.2$, and $\epsilon_{d}=0.01$ ). (a) The signal RIDB with the rectangular time and frequency windows of length $N / 4+1$. (b) Extracted first sinusoidal FM signal component. (c) Extracted linear FM signal component. (d) Extracted second sinusoidal FM signal component.

In the next stage of the modified algorithm, the two $\left(t_{0}^{\prime}, f_{0}^{\prime}\right)$ values are set as two $\left(t_{0}, f_{0}\right)$, and the above-described procedure is repeated for each of them as long as the $\left(t_{0}, f_{0}\right)$ value exceeds the threshold $\epsilon_{c}$ or until the TFD boundaries are reached. The extracted $\left(t_{0}, \mathrm{f}_{0}\right)$ values then form one signal component. Once the component is detected, it is extracted from the $(t, f)$ plane and the procedure is repeated for the next component present in the signal. The algorithm stops once the remaining energy in the TFD becomes smaller than the preset threshold $\epsilon_{d}$.

The original method, due to its single-direction search, results in components sections or parts (not whole components), thus the components classification procedure needs also to be employed in order to combine those parts into signal components.

The modified method which applies a double-direction component search (as shown in Figure 3 ) enables us to accurately and efficiently obtain whole components without having to perform any additional classification procedure based on the minimum distance between the components.

\subsection{Example of Multicomponent Signal Components Extrac-} tion. In order to illustrate the performance of the modified algorithm for signal components extraction from its RIDB, the signal mixture containing two sinusoidal FM components and a linear FM component was used (see Figure 4). Note that unlike the algorithm in [33], the modified algorithm presented in this paper does not require that all components must have same time supports. The multicomponent signal RIDB calculated with the fixed time and frequency smoothing rectangular windows, the length of which was set to $N / 4+1$ ( $N$ being the signal length), is shown in Figure 4(a). However, the adaptive window widths 
will be used for the components IF estimation in the rest of this paper, as described in Section 3.

The extracted components are shown in Figures 4(b), $4(\mathrm{c})$, and 4(d). As it can be seen, the components are well identified with their time and frequency supports being well preserved, which is necessary for their IF estimation.

\section{IF Estimation Method Based on the Improved Sliding Pairwise ICI Rule}

3.1. Review of the IF Estimation Method Based on the ICI Rule. Once the components are extracted from a multicomponent signal TFD, their IFs can be obtained as the component maxima in the time-frequency plane using some of the existing monocomponent IF estimation methods. However, estimating the IF as the TFD maxima results into estimation bias which is caused either by IF higher order derivatives, small noise (which moves the local maxima within the signal component), or high noise (which moves the local maxima outside the component) [38]. The estimation bias increases with the window length used for TFD calculation, while the variance decreases $[6,25]$. Hence, due to the tradeoff between the estimation bias and variance, the estimation error reduction can be obtained using the proper window length [25].

The adaptive method for selecting the appropriate window width based on the ICI rule was efficiently applied to monocomponent signal IF estimation in $[24,25]$. The main advantage of this estimation method is that it does not require the knowledge of estimation bias (unlike some plugin methods, e.g., [39]), but only the estimation variance (which can be easily obtained in the case of high sampling rate and white noise). This is very useful in applications such as speech and music processing, biological signals analysis, radar, sonar, and geophysical applications [25].

For this reason, we have selected this algorithm as a basis and starting point for our new proposed methodology; we briefly review the ICI algorithm below and then show the modifications that are needed in order to apply it to multicomponent signals IF estimation.

Let us now consider a discrete nonstationary multicomponent signal in additive noise

$$
y(n)=x(n)+\epsilon(n)
$$

where

$$
x(n)=\sum_{m=1}^{M} z_{m}(n)=\sum_{m=1}^{M} a_{m}(n) e^{j \phi_{m}(n)},
$$

where $M$ is the number of signal components, $\epsilon(n)$ is white complex-valued Gaussian noise with mutually independent real and imaginary zero-mean parts of variance $\sigma_{\epsilon}^{2} / 2, a_{m}(n)$ is the $m$ th component instantaneous amplitude, and $\phi_{m}(n)$ is its instantaneous phase [1].

The component IF can be estimated from the signal TFD as [1]

$$
\widehat{\omega}_{m}(n, h)=\arg \left[\max _{k} \operatorname{TFD}_{m}(n, k, h)\right]
$$

where $\operatorname{TFD}_{m}(n, k, h)$ is the TFD containing only the $m$ th component extracted from the multicomponent signal TFD calculated using the window of length $h$. It was shown in [25] that for the asymptotic case (small estimation error) the IF estimation error $\Delta \hat{\omega}_{m}(n, h)=\omega_{m}(n)-\widehat{\omega}_{m}(n, h)$ is

$$
\left|\Delta \widehat{\omega}_{m}(n, h)\right| \leq\left|\operatorname{bias}_{m}(n, h)\right|+\kappa \sigma_{m}(h),
$$

with probability $P(\kappa)$, where $\kappa$ is a quantile of the standard Gaussian distribution, and $\sigma_{m}(h)$ the component estimation error standard deviation obtained as

$$
\sigma_{m}(h)=\sqrt{\frac{\sigma_{\epsilon}^{2}}{2\left|A_{m}\right|^{2}}\left(1+\frac{\sigma_{\epsilon}^{2}}{2\left|A_{m}\right|^{2}}\right) \frac{T}{h^{3}} \frac{E}{F^{2}}},
$$

where $E=\sum_{i=1}^{h} w(i)(i / h)^{2}, F=\sum_{i=1}^{h}[w(i)]^{2}(i / h)^{2}$, and $w(n)$ is the real-valued symmetrical window of length $h$ [25]. The $E$ and $F$ values depend on the window type used for TFD calculation. For example, in the case of the rectangular window $E=F=1 / 12$ [25].

As shown in [25], for

$$
\left|\operatorname{bias}_{m}(n, h)\right| \leq \kappa \sigma_{m}(h),
$$

Equation (13) becomes

$$
\left|\Delta \hat{\omega}_{m}(n, h)\right| \leq 2 \kappa \sigma_{m}(h) .
$$

Equations (13) and (16) imply that $\omega_{m}(n)$ belongs to the confidence interval $D_{m}(n, l)=\left[L_{m}(n, l), U_{m}(n, l)\right]$ with probability $P(\kappa)$ (the larger $\kappa$ value gives $P(\kappa)$ closer to 1 ), where its upper $U_{m}(n, l)$ and lower $L_{m}(n, l)$ limits are defined as

$$
\begin{aligned}
& U_{m}(n, l)=\left[\widehat{\omega}_{m}(n, h)+2 \kappa \sigma_{m}(h)\right], \\
& L_{m}(n, l)=\left[\widehat{\omega}_{m}(n, h)-2 \kappa \sigma_{m}(h)\right],
\end{aligned}
$$

and $l$ is the sequence number of $h$ in a set of increasing window widths $H=\left\{h_{l} \mid h_{1}<h_{2}<\cdots<h_{J}\right\}$. The IF estimation method proposed in $[24,25]$ calculates a sequence of TFDs for each of the window widths from $H$. In general, any reasonable choice of $H$ is acceptable [25]. In this paper, we have used $H=\left\{h_{l} \mid h_{l}=h_{l-1}+2\right\}$, same as in [26], with $h_{1}=N / 8+1$ and $h_{J}=N / 2+1$.

Then, the components separation and extraction procedure is performed, as described in Section 2, resulting in $m$ component TFDs (denoted as $\operatorname{TFD}_{m}(n, k, h)$ ) for each $h$ from $H$.

Next, the set of $J$ IFs estimates is obtained using (12) for each of the signal components followed by the confidence intervals $D_{m}(n, l)$ calculation for each time instant $n T$ ( $T$ is the sampling interval) and each window width $h$. This adaptive method tracks the intersection of the current confidence interval $D_{m}(n, l)$ and the previous one $D_{m}(n, l-$ $1)$, giving the best window width for each time instant $n T$ as the largest one from $H$ for which it is true that $[24,25]$

$$
D_{m}(n, l-1) \cap D_{m}(n, l) \neq 0 .
$$

A justification for such an adaptive data-dependent selection of window width size independently for each time 
instant $n T$, and each signal component lays in the fact that for the confidence intervals $D_{m}(n, l-1)$ and $D_{m}(n, l)$ which do not intersect, the inequality (16) is not satisfied for at least one $h$ from $H$ [25]. This is caused by the estimation bias being too large when compared to the variance (what is contrary to the condition in inequality (15)) [25]. Thus, the largest $h$ for which (18) is satisfied is considered to give the optimal bias-to-variance tradeoff resulting in a reduced estimation error [24].

3.2. IF Estimation Method Based on the Improved Sliding Pairwise ICI Rule. In this section, the above-described algorithm for adaptive frequency smoothing window size selection is improved and modified such that it can be used in multicomponent IF estimation.

The quantile of the standard Gaussian distribution $\kappa$ value plays a crucial role in the ICI method in the proper window size calculation, and hence in estimation accuracy [40]. Various computationally demanding methods for its selection were proposed, such as the one using crossvalidation [41]. As it was shown in [42], smaller $\kappa$ values give too short window widths, while large $\kappa$ values (for which $P(\kappa) \rightarrow 1)$ result in oversized window widths, both disturbing the estimation accuracy.

One of the ways to improve the proper window width selection using the ICI rule is to track the amount of overlap between the consecutive confidence intervals (unlike the ICI method which only requires their overlap). Furthermore, as opposed to the adaptive window size selection procedure given in [40] (which demands the intersection of current confidence interval with all previous intervals in order for it to be a candidate for the finally selected window width for the considered time instant $n T$ ), this new proposed method requires only a pairwise intersection of two consecutive confidence intervals, same as in $[24,25]$.

Here, we introduce the $\mathrm{C}_{m}(n, l)$ as the amount of overlap between two consecutive confidence intervals

$$
C_{m}(n, l)=\left|D_{m}(n, l) \cap D_{m}(n, l-1)\right| .
$$

In order to have a measure of the confidence intervals overlap belonging to a finite interval, the $C_{m}(n, l)$ can be normalized with the size of the current confidence interval, defining the $O_{m}(n, l)$ as the following ratio

$$
O_{m}(n, l)=\frac{C_{m}(n, l)}{\left|D_{m}(n, l)\right|} .
$$

Thus, the $O_{m}(n, l)$ value, unlike the $C_{m}(n, l)$, always belongs to the interval $[0,1]$, making it easy to introduce the preset threshold value $O_{c}$ as an additional criterion for the most appropriate window width selection

$$
O_{m}(n, l) \geq O_{c},
$$

where

$$
O_{m}(n, l)= \begin{cases}0, & C_{m}(n, l)=0 \\ 1, & C_{m}(n, l)=\left|D_{m}(n, l)\right| \\ \langle 0,1\rangle & \text { elsewhere. }\end{cases}
$$

TABLE 1: IF estimation MAE and MSE comparison obtained using

\begin{tabular}{|c|c|c|c|c|c|c|}
\hline & \multicolumn{5}{|c|}{$20 \log \left(A / \sigma_{\epsilon}\right)$} \\
\hline & & 2 & 5 & 10 & 15 & 20 \\
\hline \multicolumn{7}{|c|}{ Component 1} \\
\hline \multirow{3}{*}{ MAE } & ICI & 23.55 & 20.71 & 14.76 & 14.16 & 13.74 \\
\hline & Imp. ICI & 22.35 & 19.31 & 14.44 & 14.04 & 13.75 \\
\hline & Imp. [\%] & 5.11 & 6.79 & 2.14 & 0.80 & -0.12 \\
\hline \multirow{3}{*}{ MSE } & ICI & 6442.9 & 5635.7 & 4170.0 & 4121.6 & 4043.5 \\
\hline & Imp. ICI & 6466.5 & 5639.8 & 4171.0 & 4121.3 & 4043.5 \\
\hline & Imp. [\%] & -0.37 & -0.07 & -0.02 & 0.01 & 0.00 \\
\hline \multicolumn{7}{|c|}{ Component 2} \\
\hline \multirow{3}{*}{ MAE } & ICI & 15.49 & 11.53 & 9.35 & 8.76 & 8.44 \\
\hline & Imp. ICI & 15.28 & 11.36 & 9.27 & 8.75 & 8.44 \\
\hline & Imp. [\%] & 1.37 & 1.51 & 0.92 & 0.17 & 0.00 \\
\hline \multirow{3}{*}{ MSE } & ICI & 3352.9 & 2621.9 & 2216.8 & 2113.6 & 2065.8 \\
\hline & Imp. ICI & 3344.1 & 2616.9 & 2215.9 & 2113.6 & 2065.8 \\
\hline & Imp. [\%] & 0.26 & 0.19 & 0.04 & 0.00 & 0.00 \\
\hline \multicolumn{7}{|c|}{ Component 3} \\
\hline \multirow{3}{*}{ MAE } & ICI & 7.56 & 6.29 & 4.90 & 4.44 & 4.35 \\
\hline & Imp. ICI & 7.04 & 5.71 & 4.77 & 4.43 & 4.34 \\
\hline & Imp. [\%] & 6.89 & 9.14 & 2.73 & 0.39 & 0.29 \\
\hline \multirow{3}{*}{ MSE } & ICI & 404.0 & 284.2 & 220.4 & 204.3 & 203.9 \\
\hline & Imp. ICI & 394.8 & 275.9 & 219.2 & 204.3 & 203.9 \\
\hline & Imp. [\%] & 2.26 & 2.91 & 0.54 & 0.00 & 0.00 \\
\hline
\end{tabular}
the MBD for methods based on the ICI and improved ICI rule for the signal $x_{1}(n)\left(\beta=0.1, \kappa=1.75, O_{c}=0.97, \epsilon_{c}=0.2, \epsilon_{d}=0.01\right.$, adaptive rectangular time, and lag windows).

This additional criterion defined in (21) sets more strict requirements for the window width selection (when compared to the ICI rule which requires only the intersection of confidence intervals and does not consider the amount of their intersection), reducing the estimation inaccuracy by preventing oversized window widths selection, as it was shown in $[26,42]$.

Unlike the monocomponent IF estimation methods in [24-26], the multicomponent IF estimation method proposed in this paper combines the modified component extraction method with the above-described improved ICI rule. The method proposed in [6], however, is based on the original ICI rule and an unmodified component tracking algorithm. Apart from a set of the IF estimates calculated with fixed-size frequency smoothing window widths, this improved adaptive algorithm based on the improved ICI rule was then used to select the best IF estimate for each time instant. The method results in enhanced components IF estimation accuracy in terms of both mean absolute error (MAE) and mean squared error (MSE) for various SNRs and different window types when compared to the ICI method, as it is shown in the Section 4.

3.3. Summary of the Newly Proposed Multicomponent IF Estimation Method. Before we illustrate the use of the proposed algorithm on several examples, we will first summarize 
TABLE 2: IF estimation MAE and MSE comparison obtained using the RIDB for methods based on the ICI and improved ICI rule for the signal $x_{1}(n)\left(\kappa=1.75, O_{c}=0.97, \epsilon_{c}=0.2, \epsilon_{d}=\right.$ 0.01 , rectangular time smoothing window of size $N / 4+1$, adaptive rectangular frequency smoothing window).

\begin{tabular}{|c|c|c|c|c|c|c|}
\hline & & \multicolumn{5}{|c|}{$\left.20 \log \left(A / \sigma_{\epsilon}\right)\right)$} \\
\hline & & 2 & 5 & 10 & 15 & 20 \\
\hline \multicolumn{7}{|c|}{ Component 1} \\
\hline \multirow{3}{*}{ MAE } & ICI & 11.52 & 10.38 & 7.36 & 5.31 & 4.13 \\
\hline & Imp. ICI & 8.23 & 7.80 & 5.68 & 4.84 & 4.10 \\
\hline & Imp. [\%] & 28.60 & 24.85 & 22.81 & 8.77 & 0.87 \\
\hline \multirow{3}{*}{ MSE } & ICI & 1586.7 & 1591.5 & 882.4 & 670.0 & 426.5 \\
\hline & Imp. ICI & 1512.8 & 1548.9 & 863.4 & 666.6 & 426.8 \\
\hline & Imp. [\%] & 4.66 & 2.67 & 2.15 & 0.50 & -0.07 \\
\hline \multicolumn{7}{|c|}{ Component 2} \\
\hline \multirow{3}{*}{ MAE } & ICI & 7.00 & 5.82 & 4.75 & 4.12 & 3.90 \\
\hline & Imp. ICI & 6.05 & 5.12 & 4.44 & 4.08 & 3.89 \\
\hline & Imp. [\%] & 13.61 & 11.95 & 6.50 & 0.94 & 0.38 \\
\hline \multirow{3}{*}{ MSE } & ICI & 210.8 & 166.3 & 110.4 & 85.6 & 74.6 \\
\hline & Imp. ICI & 166.7 & 136.4 & 103.6 & 85.2 & 74.5 \\
\hline & Imp. [\%] & 20.92 & 18.00 & 6.20 & 0.45 & 0.10 \\
\hline \multicolumn{7}{|c|}{ Component 3} \\
\hline \multirow{3}{*}{ MAE } & ICI & 5.64 & 5.40 & 4.22 & 3.96 & 3.95 \\
\hline & Imp. ICI & 4.10 & 3.91 & 3.69 & 3.83 & 3.93 \\
\hline & Imp. [\%] & 27.29 & 27.73 & 12.59 & 3.16 & 0.47 \\
\hline \multirow{3}{*}{ MSE } & ICI & 162.8 & 185.8 & 193.8 & 244.1 & 265.0 \\
\hline & Imp. ICI & 136.5 & 161.5 & 187.8 & 242.7 & 264.8 \\
\hline & Imp. [\%] & 16.17 & 13.10 & 3.11 & 0.56 & 0.06 \\
\hline
\end{tabular}

the key steps of our newly proposed multicomponent IF estimation method.

Step 1. Calculate a set of RIDs for various frequency smoothing window lengths.

Step 2. Extract the signal components from each RID using the method described in Section 2.3.

Step 3. For each component, calculate its IF using (12).

Step 4. For each time instant and each component, choose the best IF estimate from the set of estimates calculated for different frequency smoothing window lengths using the multicomponent IF estimation method based on the improved ICI rule presented in Section 3.2.

As it is shown in Section 4, a significant IF estimation accuracy enhancement has been achieved (especially in low SNRs environments) by combining the proposed components extraction procedure with the improved ICI rule.

\section{Multicomponent IF Estimation Simulation Results}

This section gives the results obtained by the proposed multicomponent IF estimation method for two multicomponent signals of the form in (11): a three component signal
TABLE 3: IF estimation MAE and MSE comparison obtained using the RIDB for methods based on the ICI and improved ICI rule for the signal $x_{1}(n)\left(20 \log \left(A / \sigma_{\epsilon}\right)=10, \kappa=1.75, O_{c}=0.97, \epsilon_{c}=\right.$ $0.2, \epsilon_{d}=0.01$, time smoothing window of size $N / 4+1$, adaptive frequency smoothing window).

\begin{tabular}{lcccccc}
\hline & \multicolumn{5}{c}{ MAE } & MSE \\
& ICI & Imp. ICI Imp. [\%] & ICI & Imp. ICI Imp. [\%] \\
\hline Rectangular & 7.36 & 5.68 & 22.81 & 882.4 & 863.4 & 2.15 \\
Hamming & 7.61 & 6.67 & 12.39 & 1060.5 & 1046.4 & 1.32 \\
Hanning & 8.31 & 7.59 & 8.59 & 1213.7 & 1198.9 & 1.22 \\
Triangular & 8.24 & 7.27 & 11.82 & 1061.9 & 1051.9 & 0.94 \\
Gauss & 8.74 & 9.72 & -11.27 & 1112.8 & 1110.0 & 0.24 \\
\hline \multicolumn{7}{c}{ Component 2 } \\
\hline Rectangular & 4.75 & 4.44 & 6.50 & 110.4 & 103.6 & 6.20 \\
Hamming & 4.42 & 3.81 & 13.95 & 104.1 & 83.9 & 19.46 \\
Hanning & 4.51 & 3.70 & 18.00 & 114.1 & 87.6 & 23.23 \\
Triangular & 3.97 & 3.57 & 10.05 & 74.3 & 65.7 & 11.61 \\
Gauss & 4.79 & 4.36 & 9.02 & 163.2 & 139.4 & 14.59 \\
\hline \multicolumn{7}{c}{ Component 3 } \\
\hline Rectangular & 4.22 & 3.69 & 12.59 & 193.8 & 187.8 & 3.11 \\
Hamming & 4.36 & 3.72 & 14.65 & 170.2 & 167.0 & 1.85 \\
Hanning & 4.31 & 3.70 & 14.18 & 148.3 & 143.7 & 3.14 \\
Triangular & 5.20 & 4.49 & 13.72 & 324.3 & 314.3 & 3.09 \\
Gauss & 5.32 & 5.95 & -11.85 & 311.5 & 332.2 & -6.64 \\
\hline
\end{tabular}

with components of equal amplitudes $x_{1}(n)=z_{1}(n)+$ $z_{2}(n)+z_{3}(n)$, where $z_{m}(n)=A_{m} \exp \left(j \phi_{m}(n)\right)\left(A_{m}=1\right)$, and the echolocation sound emitted by a bat signal, $x_{2}(n)$, with components of different amplitudes. The achieved estimation error reduction in terms of MAE and MSE is compared to the ICI-based IF estimation method for various window types and different noise levels (defined as $\left.20 \log \left(A / \sigma_{\epsilon}\right)[25]\right)$.

The signal $x_{1}(n)$ (of length $N=128$ ) contains two sinusoidal FM components and one linear FM component with different time supports (which partially overlap); the IF law of each component is $\omega_{1}(n)=0.35+0.05 \cos (2 \pi(n-$ $\left.\left.N_{1} / 2\right) / N_{1}-\pi / 2\right), \omega_{2}(n)=0.05+0.3(n-1) /\left(N_{2}-1\right)$, and $\omega_{3}(n)=0.075+0.025 \cos \left(2 \pi\left(n-N_{3} / 2\right) / N_{3}-\pi / 2\right)$. The component lengths are $N_{1}=96, N_{2}=48$, and $N_{3}=48$.

The TFDs we have used are the MBD and the RID defined in (7) and (8), respectively, calculated and plotted using the Time-Frequency Signal Analysis Toolbox (see Article 6.5 in [1] for more details), with varying frequency smoothing window lengths belonging to the set $H$ which contains 25 increasing window lengths, the time smoothing window length is $N / 4+1$ (found to be, based on extensive simulations, a suitable choice for broad classes of signals), and the number of frequency bins $N_{f}=4 \mathrm{~N}$. The component separation and extraction procedure was done using $\Delta f=F / 2=N_{f} / 8$, $\epsilon_{c}=0.2$, and $\epsilon_{d}=0.01$. The parameter $\kappa$ value used in both IF estimation methods, based on the ICI and the improved ICI rule, was set to $\kappa=1.75$ (as in $[24,25]$ ). Based 


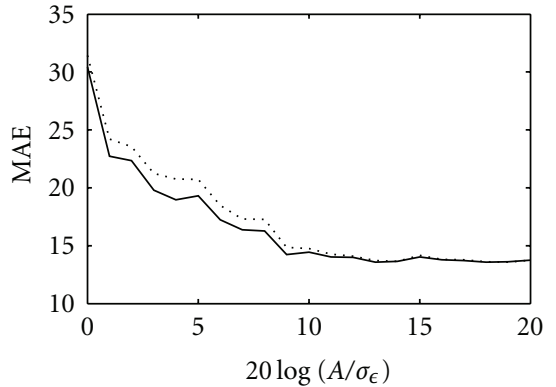

…. ICI
- Imp. ICI

(a)

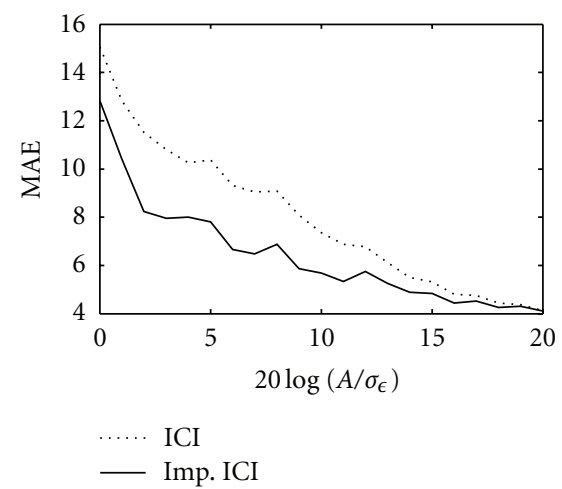

(d)

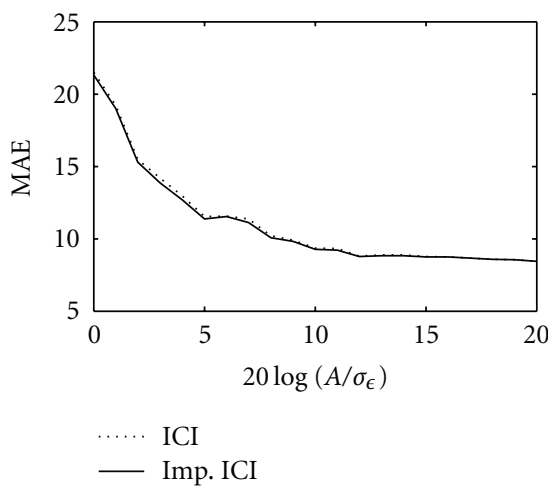

(b)

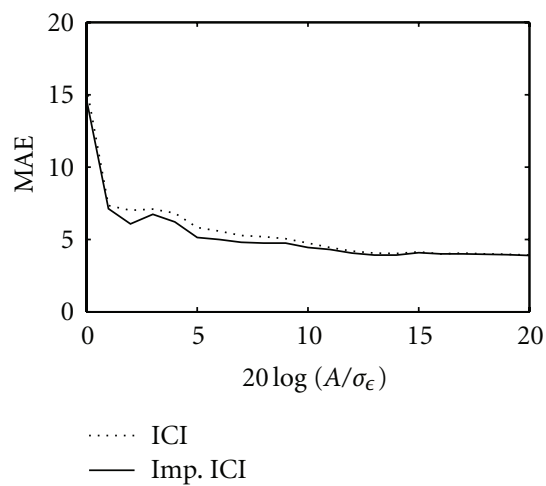

(e)

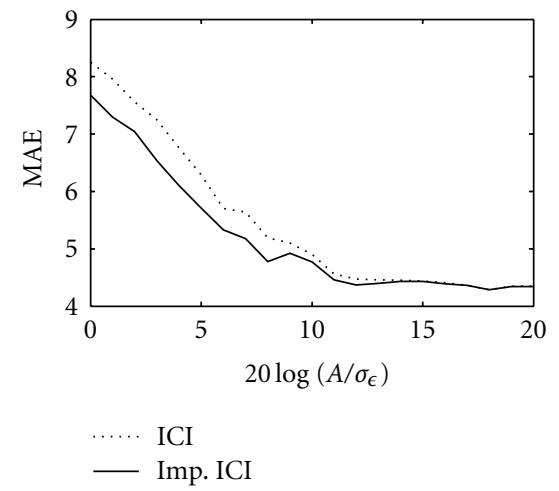

(c)

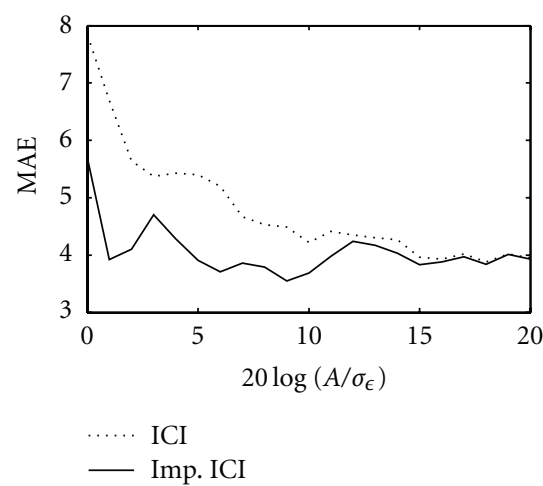

(f)

FIGURE 5: IF estimation MAE over a range of noise levels using the methods based on the ICI and the improved ICI rule for the signal $x_{1}(n)$ $\left(\kappa=1.75, O_{c}=0.97, \epsilon_{c}=0.2, \epsilon_{d}=0.01\right)$. (a) First component IF MAE obtained using the MBD. (b) Second component IF MAE obtained using the MBD. (c) Third component IF MAE obtained using the MBD. (d) First component IF MAE obtained using the RIDB. (e) Second component IF MAE obtained using the RIDB. (f) Third component IF MAE obtained using the RIDB.

on numerous simulations performed on various classes of signals, the threshold $O_{c}=0.97$ was shown to result in the largest estimation error reduction, as shown in [26].

Tables 1 and 2 show, respectively, that the IF estimation MAE and MSE (averaged over 100 Monte Carlo simulations runs) for the ICI and the improved ICI-based method using both the MBD and the RIDB with the rectangular time and frequency smoothing windows for different noise levels $20 \log \left(A / \sigma_{\epsilon}\right)=[2,5,10,15,20]$. As it can be seen from the Tables 1 and 2, the RIDB was shown to be more robust for IF estimation from multicomponent signals in additive noise, outperforming the estimation error reduction results achieved by using the MBD. Furthermore, the largest MAE and MSE improvement for each component was obtained for the low SNR while for the higher SNRs both methods perform almost identically. This MAE improvement using the improved ICI method when compared to the ICI-based method varies from around $1 \%$ to $28 \%$ while the MSE reduction goes from around $0 \%$ to $23 \%$. As the IF estimation of signals for low SNRs is much more complex than in the case of high SNRs, the improvements in estimation error reduction using this new proposed method show the strength of the method over other similar approaches [43]. The same conclusion can be drawn from Figure 5 which shows the IF estimation MAE as a function of the noise intensity for both the ICI-based and the improved ICI-based method.

Table 3 gives the MAE and MSE results obtained using the ICI method and its modification proposed in this paper for $20 \log \left(A / \sigma_{\epsilon}\right)=10$ and different window types (rectangular, Hamming, Hanning, triangular, and Gauss). As it can be seen, the improved ICI-based method results in reduced MAEs by up to $22 \%$ and MSE reduced by up to $23 \%$.

The noisy three component signal $x_{1}(n)$ in additive noise $\left(20 \log \left(A / \sigma_{\epsilon}\right)=10\right)$ is shown in Figure 6(a) while its magnitude and phase spectrum is given in Figure 6(b). The magnitude and phase spectra give information of the signal frequency content, but not the times when certain frequencies are present in the signal. This information can be obtained from the signal TFD. The signal time-frequency representation using the RIDB with rectangular frequency smoothing windows of the fixed lengths $h_{1}=N / 8+1$ and $h_{25}=N / 2+1$, and its corresponding IFs $\hat{\omega}_{m}\left(n, h_{1}\right)$ and $\hat{\omega}_{m}\left(n, h_{25}\right)$ calculated using (12) are shown in Figures 6(c), 6(d), 6(e), and 6(f), respectively. The IFs estimated using the ICI and improved ICI-based methods are, respectively, given in Figures $6(\mathrm{~g})$ and $6(\mathrm{~h})$. The IF estimation error 


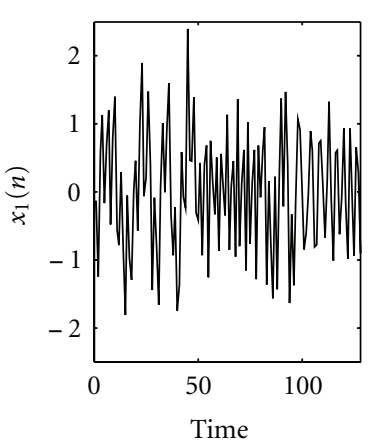

(a)

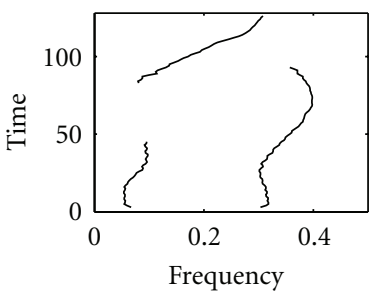

(e)

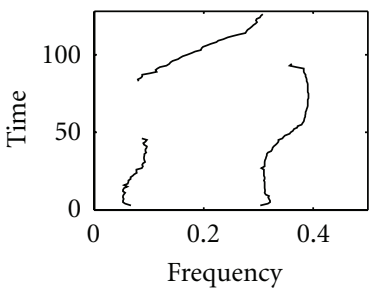

(g)
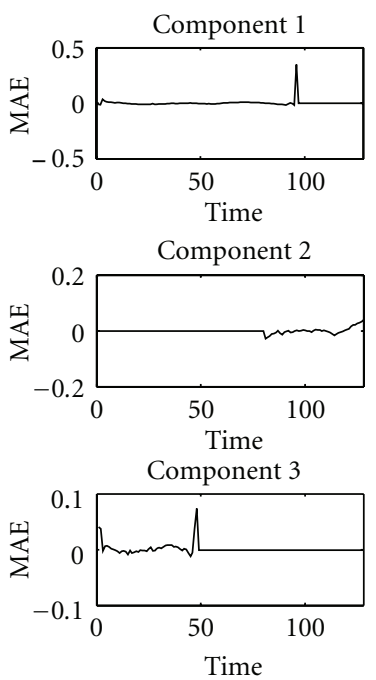

(k)
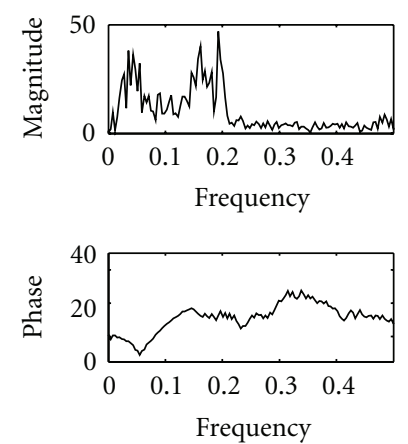

(b)

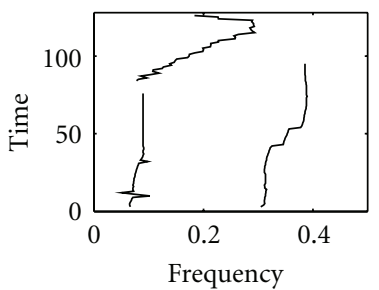

(f)

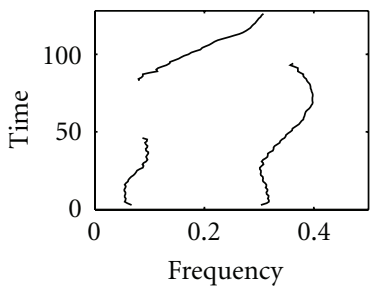

(h)
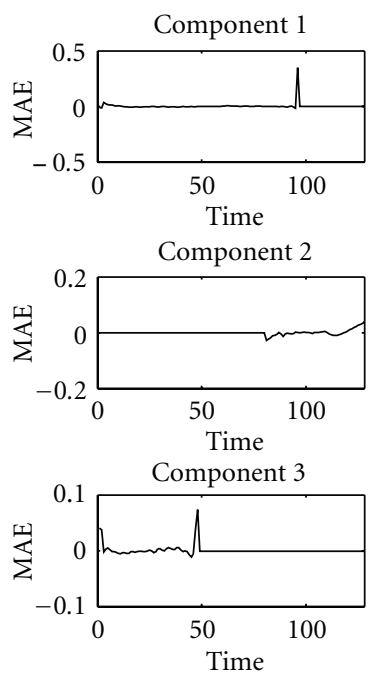

(1)

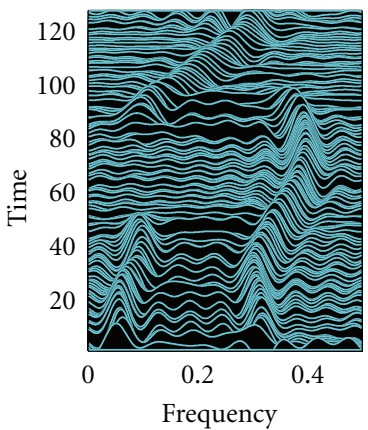

(c)

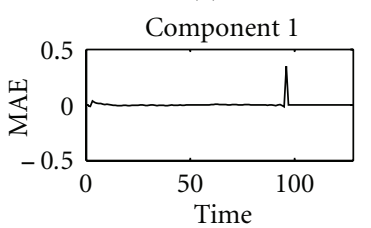

Component 2
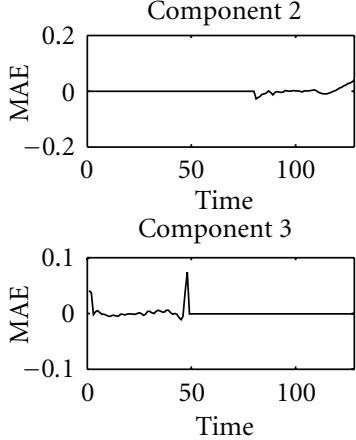

(i)
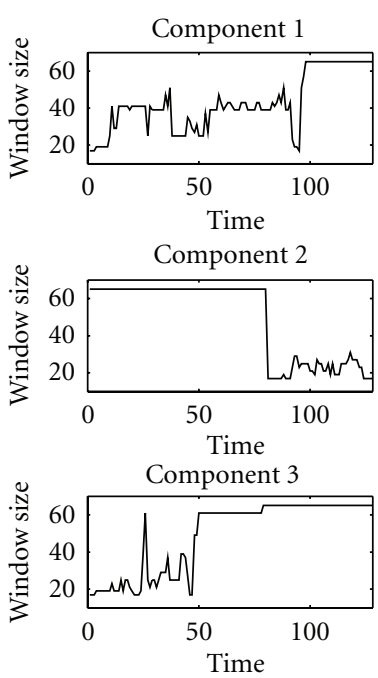

(m)

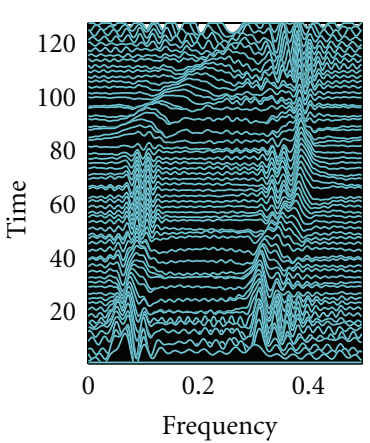

(d)
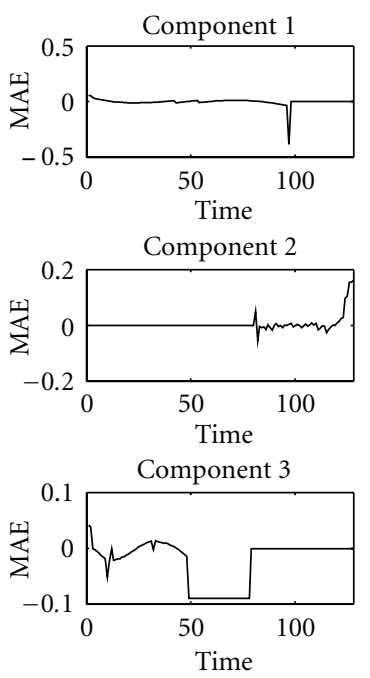

(j)
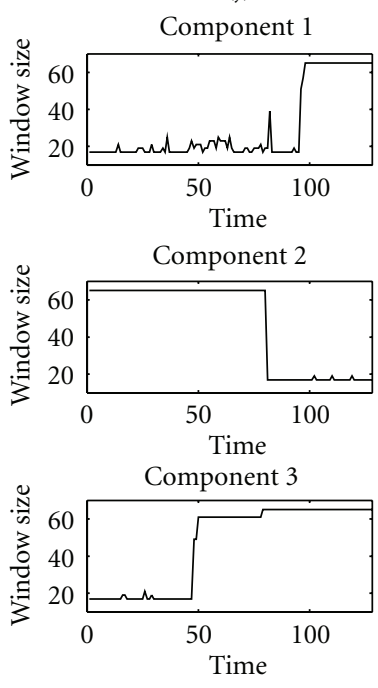

(n)

FIgURE 6: (a) Noisy multicomponent signal $x_{1}(n)$ in time $\left(20 \log \left(A / \sigma_{\epsilon}\right)=10\right)$. (b) Signal magnitude and phase spectrum. (c) The signal RIDB for $h_{1}$. (d) The signal RIDB for $h_{25}$. (e) Estimated IFs for $h_{1}$. (f) Estimated IFs for $h_{25}$. (g) Estimated IFs using the ICI-based method. (h) Estimated IFs using the improved ICI-based method. (i) IFs estimation MAE for $h_{1}$. (j) IFs estimation MAE for $h_{25}$. (k) IFs estimation MAE using the ICI-based method. (l) IFs estimation MAE using the improved ICI-based method. (m) Components window size for the ICI-based method. (n) Components window size for the improved ICI-based method. 


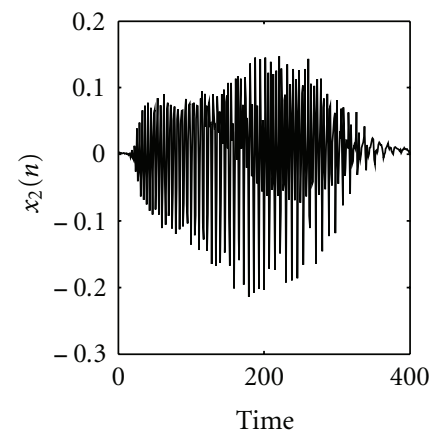

(a)

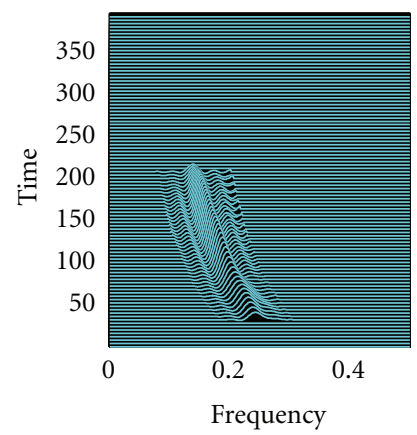

(e)

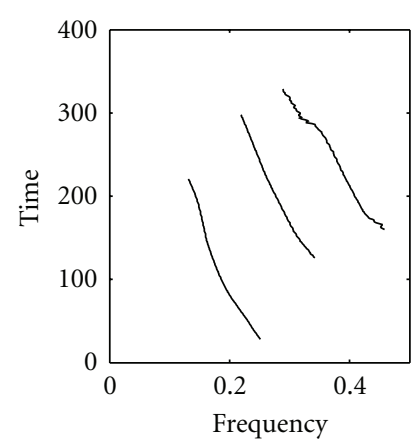

(i)

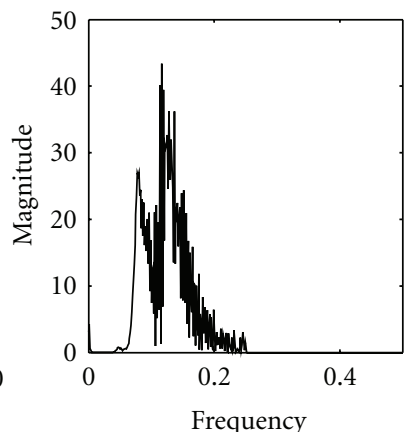

(b)

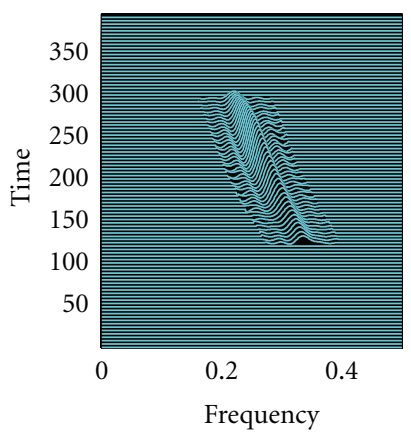

(f)
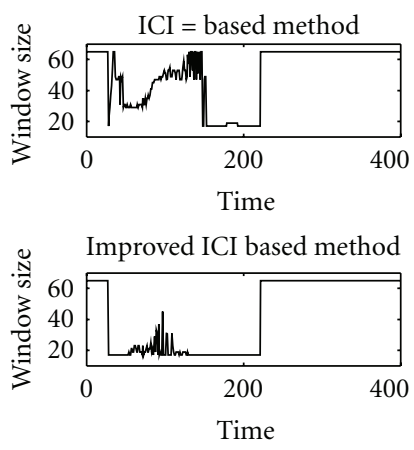

(j)

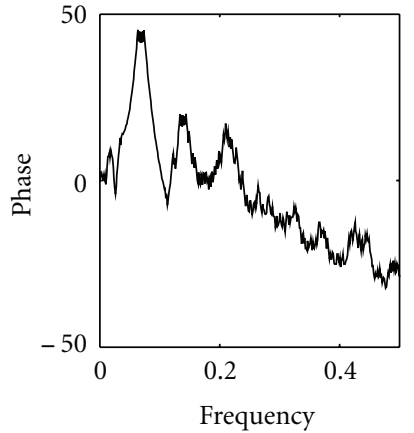

(c)

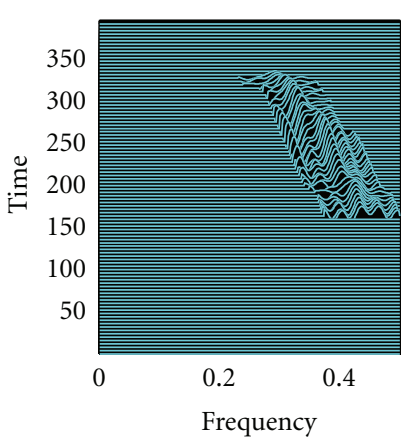

(g)
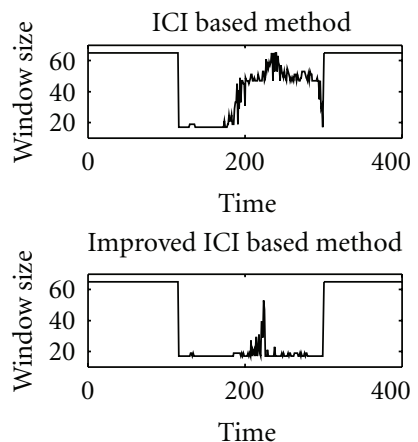

(k)

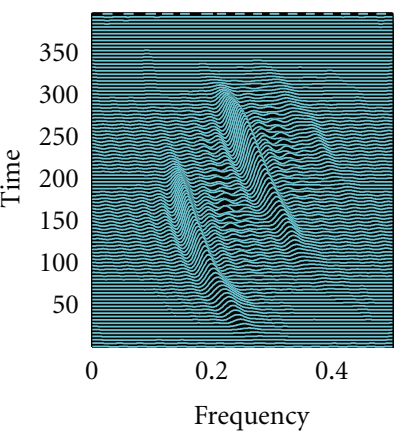

(d)

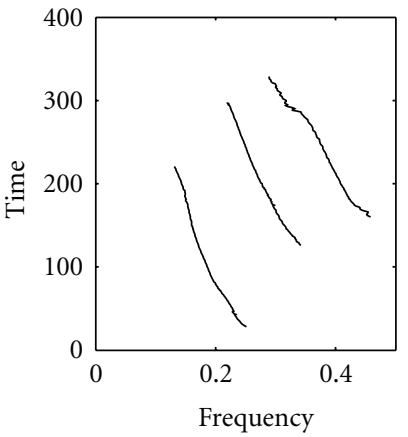

(h)
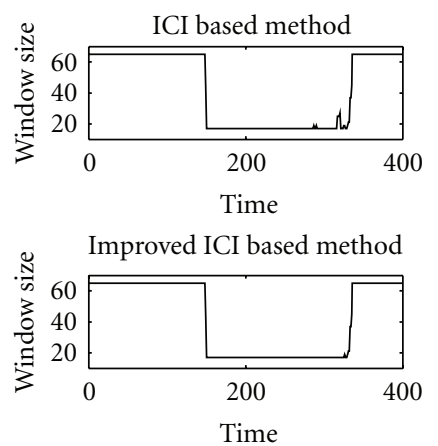

(1)

FIgURE 7: (a) The bat signal $x_{2}(n)$ in time. (b) The bat signal magnitude spectrum. (c) The bat signal phase spectrum. (d) The RIDB of the bat signal for fixed-size rectangular time smoothing window $h=N / 4+1$. (e) The bat signal first component. (f) The bat signal second component. (g) The bat signal third component. (h) IFs of the bat signal components obtained using the ICI-based method. (i) IFs of the bat signal components obtained using the improved ICI-based method. (j) Window size for the first component (obtained by the ICI-based and improved ICI-based method). (k) Window size for the second component (obtained by the ICI-based and improved ICI-based method). (l) Window size for the third component (obtained by the ICI-based and improved ICI-based method).

of each component is shown in Figures 6(i), 6(j), 6(k), and 6(l). Finally, the window size used by the ICI and the improved ICI-based method for each component as a function of time is given in Figures 6(m) and 6(n). As it can be seen, the improved ICI rule, due to its more strict criterion in the window size selection, uses shorter window widths when compared to the ICI rule, resulting in a significant IF estimation accuracy improvement.

Figures 7(a), 7(b), 7(c), and 7(d) show the bat echolocation signal in time, its magnitude spectra, phase spectra, and the RID with the fixed-size rectangular time smoothing window $h=33$, respectively. The extracted signal components using the modified method described in Section 4 are shown in Figures $7(\mathrm{e}), 7(\mathrm{f})$, and $7(\mathrm{~g})$, which show that each component is correctly detected and extracted, followed by the components IF estimation. The estimated IFs using the ICI and the improved ICI-based method are shown in Figures $7(\mathrm{~h})$ and $7(\mathrm{i})$. The adaptive varying window sizes used by the two methods are given in Figures $7(\mathrm{j}), 7(\mathrm{k})$, and 7 (l) for each component, respectively.

A comparison of the noisy multicomponent signal $x_{1}(n) \mathrm{MBD}, \mathrm{RIDB}$, and the TFD reconstructed from the components IFs estimated using the improved ICI-based method is given in Figure 8. It can be observed, that once the components are extracted and their IF laws are estimated using the proposed method, we are able to get a cross-terms 


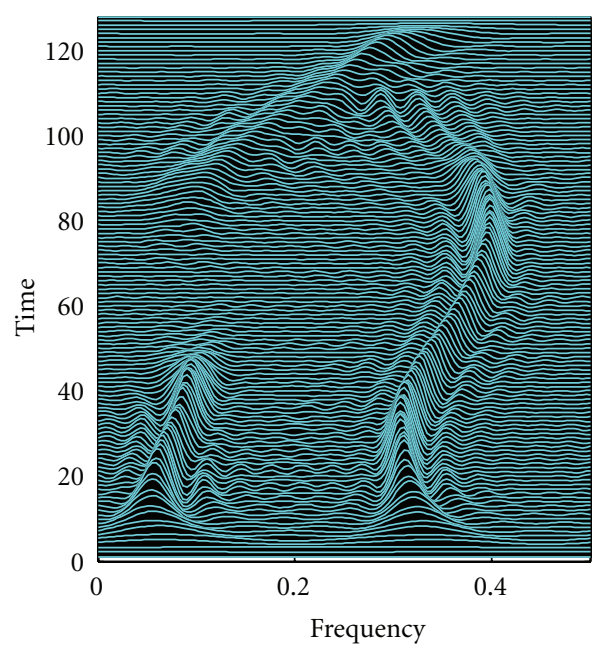

(a)

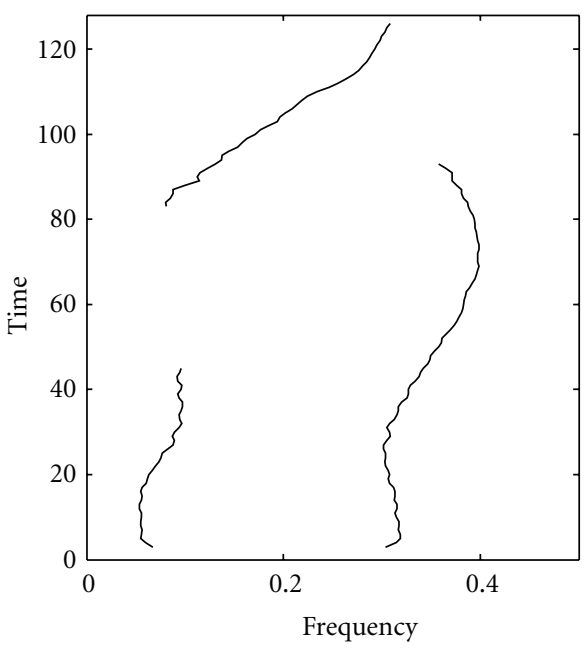

(c)

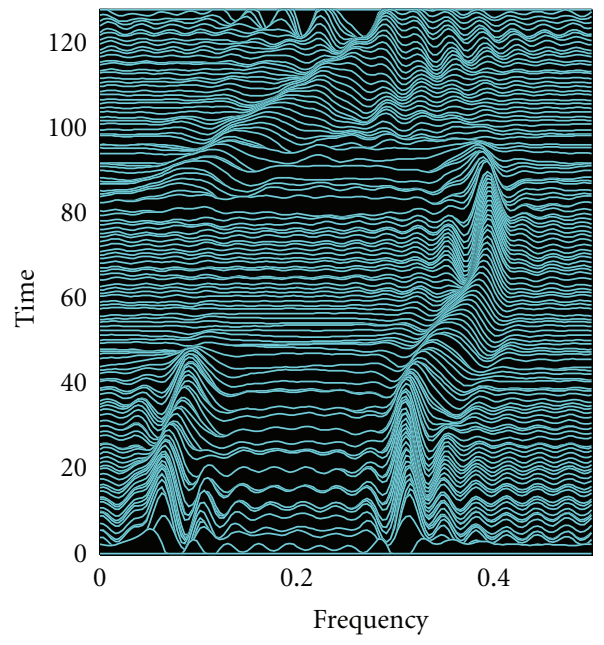

(b)

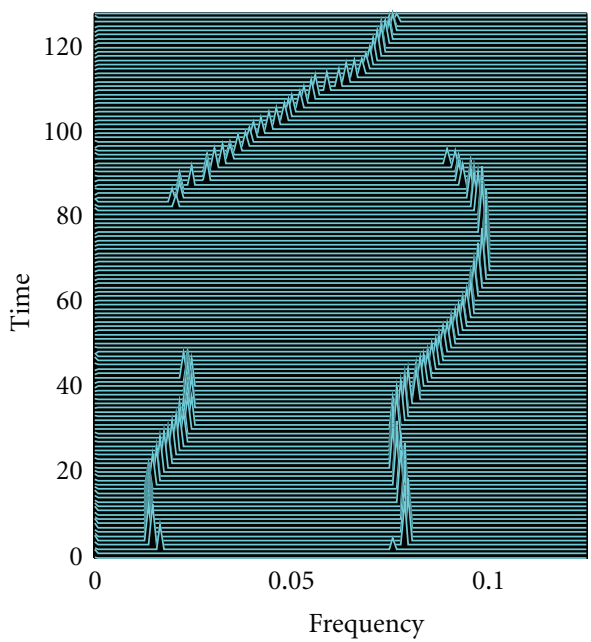

(d)

FIGURE 8: (a) Noisy multicomponent signal $x_{1}(n)$ MBD with rectangular time and lag window of length $N / 4+1\left(20 \log \left(A / \sigma_{\epsilon}\right)=10\right)$. $(\mathrm{b})$ The signal RIDB with rectangular time and frequency smoothing windows of length $N / 4+1$. (c) The noisy signal components IFs obtained using the improved ICI-based method. (d) The signal reconstructed TFD from the estimated components IFs obtained using the improved ICI-based method.

free and high time-frequency resolution multicomponent signal TFD, shown in Figure 8(d).

The bat echolocation signal $x_{2}(n) \mathrm{MBD}, \mathrm{RIDB}$, and the TFD reconstructed from the components IFs estimated using the method described in Section 3.2 is given in Figure 9. As for the signal $x_{1}(n)$, a cross-terms free and high resolution TFD is again obtained from the estimated components IFs.

\section{Conclusion}

A novel multicomponent signal instantaneous frequency (IF) estimation method has been presented. A modification of the blind (i.e., without a priori information) components separation method for separation and extraction of components from a noisy signal mixture was combined with the IF estimation method based on the improved intersection of confidence intervals (ICI) rule. This new method was compared to the ICI-based IF estimation method, demonstrating significant IF estimation quality improvements in terms of the mean absolute error (MAE) and the mean squared error (MSE) reduction in spite of the artifacts present in the time-frequency distribution of the analyzed noisy nonstationary signal. The new method's performance was analyzed for different signal-to-noise ratios (SNRs) and various window types used in the reduced interference distribution calculation, resulting in an estimation error reduction in terms of the MAE by up to $28 \%$ and the MSE reduction by up to $23 \%$ when compared to the unmodified ICI-based method. Furthermore, the best results have been achieved for low SNRs, making the proposed method an efficient technique for multicomponent signals IF estimation in high-noise environments, when other similar existing approaches are known to fail. This new proposed method can be applied to the IF estimation of fast varying 


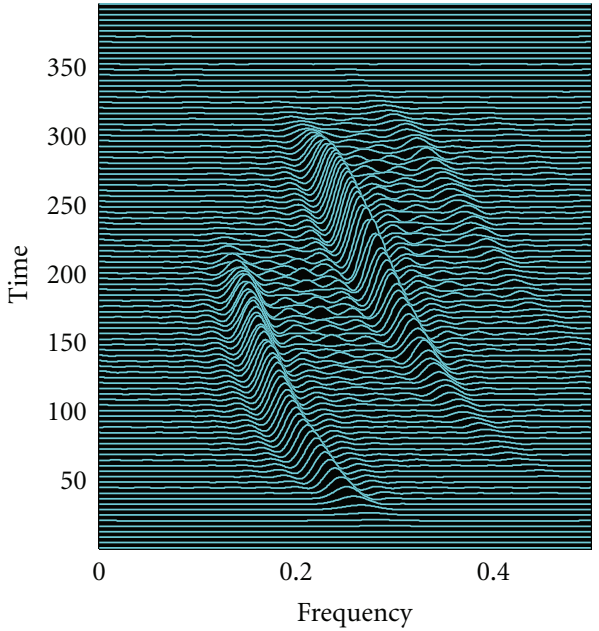

(a)

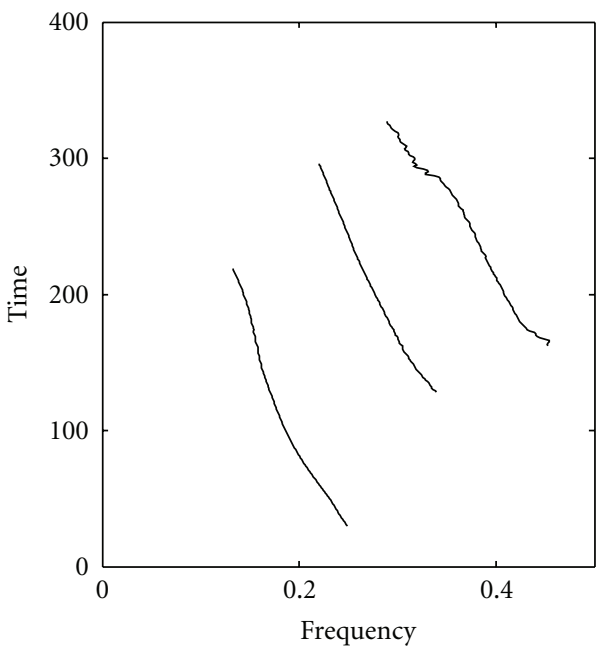

(c)

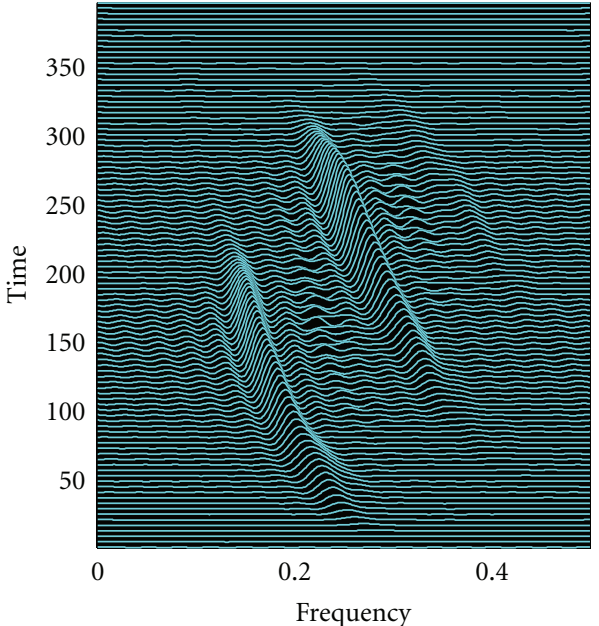

(b)

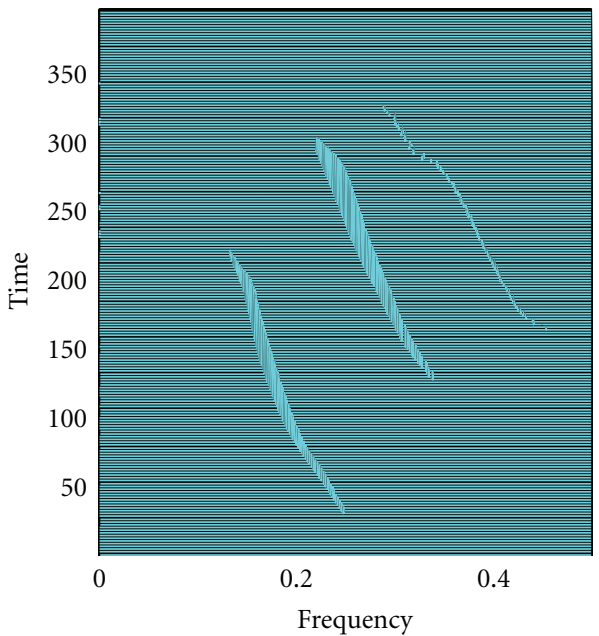

(d)

Figure 9: (a) The bat echolocation sound signal $x_{2}(n)$ MBD with rectangular time and lag window of length N/4 + 1. (b) The signal RIDB with the rectangular time and frequency smoothing windows of length $N / 4+1$. (c) The signal components IFs obtained using the improved ICI-based method. (d) The signal reconstructed TFD from the estimated components IFs obtained using the improved ICI-based method.

frequency-modulated multicomponent signals in low SNR, as illustrated by the examples presented in this paper.

\section{Acknowledgments}

This paper is a part of the research project "Optimization and Design of Time-Frequency Distributions," no. 069-03622141575 , which is financially supported by the Ministry of Science, Education, and Sports of the Republic of Croatia. This paper was also partly funded by the Qatar National Research Fund, Grant reference no. NRPR 09-626-2-243, and the Australian Research Council.

\section{References}

[1] B. Boashash, Ed., Time Frequency Signal Analysis and Processing: Acomprehensive Reference, Elsevier, The Boulevard, Langford Lane, Kidlington, Oxford, UK, 2003.
[2] B. Boashash, "Estimating and interpreting the instantaneous frequencyof a signal. I. Fundamentals," Proceedings of the IEEE, vol. 80, no. 4, pp. 520-538, 1992.

[3] B. Boashash, "Estimating and interpreting the instantaneous frequency of asignal. II. Algorithms and applications," Proceedings of the IEEE, vol. 80, no. 4, pp. 540-568, 1992.

[4] E. Bedrosian, "A product theorem for Hilbert transforms," Proceedingsof the IEEE, vol. 51, no. 5, pp. 868-869, 1963.

[5] B. Picinbono, "On instantaneous amplitude and phase of signals," IEEE Transactions on Signal Processing, vol. 45, no. 3, pp. 552-560, 1997.

[6] Z. M. Hussain and B. Boashash, "Adaptive instantaneous frequency estimation of multicomponent FM signals using quadratic time-frequency distributions," IEEE Transactions on Signal Processing, vol. 50, no. 8, pp. 1866-1876, 2002.

[7] Z. Guo, L. G. Durand, and H. C. Lee, "Time-frequency distributions of nonstationary signals based on a Bessel kernel," IEEE Transactions on Signal Processing, vol. 42, no. 7, pp. 1700-1706, 1994. 
[8] B. Boashash and V. Sucic, "Resolution measure criteria for the objective assessment of the performance of quadratic time-frequency distributions," IEEE Transactions on Signal Processing, vol. 51, no. 5, pp. 1253-1263, 2003.

[9] A. Belouchrani and M. G. Amin, "Blind source separation based on time-frequency signal representations," IEEE Transactions on Signal Processing, vol. 46, no. 11, pp. 2888-2897, 1998.

[10] Y. Zhou and B. Xu, "Blind source separation in frequency domain,” Signal Processing, vol. 83, no. 9, pp. 2037-2046, 2003.

[11] H. L. N. Thi and C. Jutten, "Blind source separation for convolutive mixtures," Signal Processing, vol. 45, no. 2, pp. 209-229, 1995.

[12] P. Smaragdis, "Blind separation of convolved mixtures in the frequency domain," Neurocomputing, vol. 22, no. 1-3, pp. 2134, 1998.

[13] D. Yellin and E. Weinstein, "Criteria for multichannel signal separation," IEEE Transactions on Signal Processing, vol. 42, no. 8, pp. 2158-2167, 1994.

[14] N. Delfosse and P. Loubaton, "Adaptive blind separation of independent sources: a deflation approach," Signal Processing, vol. 45, no. 1, pp. 59-83, 1995.

[15] Y. Inouye and K. Hirano, "Cumulant-based blind identification of linear multi-input-multi-output systems driven by colored inputs," IEEE Transactions on Signal Processing, vol. 45, no. 6, pp. 1543-1552, 1997.

[16] M. K. Emresoy and A. El-Jaroudi, "Iterative instantaneous frequency estimation and adaptive matched spectrogram," Signal Processing, vol. 64, no. 2, pp. 157-165, 1998.

[17] M. R. Morelande, B. Barkat, and A. M. Zoubir, "Statistical performance comparison of a parametric and a non-parametric method for if estimation of random amplitude linear FM signals in additive noise," in Proceedings of the 10th IEEE Signal Processing Workshop on Statistical Signal and Array Processing (SSAP '00), pp. 262-266, Pennsylvania, Pa, USA, 2000.

[18] B. Barkat and B. Boashash, "Instantaneous frequency estimation of polynomial FM signals using the peak of the PWVD: statistical performance in the presence of additive Gaussian noise," IEEE Transactions on Signal Processing, vol. 47, no. 9, pp. 2480-2490, 1999.

[19] B. Boashash and P. O'Shea, "Polynomial Wigner-Ville distributions and their relationship to time-varying higher order spectra," IEEE Transactions on Signal Processing, vol. 42, no. 1, pp. 216-220, 1994.

[20] B. Ristic and B. Boashash, "Instantaneous frequency estimation of quadratic and cubic fm signals using the cross polynomial wigner-ville distribution," IEEE Transactions on Signal Processing, vol. 44, no. 6, pp. 1549-1553, 1996.

[21] B. Porat and B. Friedlander, "Asymptotic statistical analysis of the highorder ambiguity function for parameter estimation of polynomial-phase signals," IEEE Transactions on Information Theory, vol. 42, no. 3, pp. 995-1001, 1996.

[22] L. Stanković, M. Daković, J. Jiang, and E. Sejdic, "Instantaneous frequency estimation using the $S$-transform," IEEE Signal Processing Letters, vol. 15, pp. 309-312, 2008.

[23] P. L. Shui, H. Y. Shang, and Y. B. Zhao, "Instantaneous frequency estimation based on directionally smoothed pseudoWigner-Ville distribution bank," IET Radar, Sonar and Navigation, vol. 1, no. 4, pp. 317-325, 2007.

[24] L. Stanković and V. Katkovnik, "Algorithm for the instantaneous frequency estimation using time-frequency distributions with adaptive window width," IEEE Signal Processing Letters, vol. 5, no. 9, pp. 224-227, 1998.
[25] V. Katkovnik and L. Stankovic, "Instantaneous frequency estimation using the wigner distribution with varying and data-driven window length," IEEE Transactions on Signal Processing, vol. 46, no. 9, pp. 2315-2325, 1998.

[26] J. Lerga and V. Sucic, "Nonlinear IF estimation based on the pseudo WVD adapted using the improved sliding pairwise ICI rule," IEEE Signal Processing Letters, vol. 16, no. 11, pp. 953956, 2009.

[27] H. I. Choi and W. J. Williams, "Improved time-frequency representation of multicomponent signals using exponential kernels," IEEE Transactions on Acoustics, Speech, and Signal Processing, vol. 37, no. 6, pp. 862-871, 1989.

[28] M. G. Amin and W. J. Williams, "High spectral resolution time-frequency distribution kernels," IEEE Transactions on Signal Processing, vol. 46, no. 10, pp. 2796-2804, 1998.

[29] L. Stanković, "On the realization of the polynomial wignerville distribution for multicomponent signals," IEEE Signal Processing Letters, vol. 5, no. 7, pp. 157-159, 1998.

[30] L. Rankine, M. Mesbah, and B. Boashash, "IF estimation for multicomponent signals using image processing techniques in the time-frequency domain," Signal Processing, vol. 87, no. 6, pp. 1234-1250, 2007.

[31] Z. Guo, L. G. Durand, and H. C. Lee, "Comparison of timefrequency distribution techniques for analysis of simulated Doppler ultrasound signals of the femoral artery," IEEE Transactions on Biomedical Engineering, vol. 41, no. 4, pp. 332342, 1994.

[32] S. Kodituwakku, T. D. Abhayapala, and R. A. Kennedy, "Atrial fibrillation analysis using Bessel kernel based time frequency distribution technique," in Computers in Cardiology, pp. 837840, September 2008.

[33] B. Barkat and K. Abed-Meraim, "Algorithms for blind components separation and extraction from the time-frequency distribution of their mixture," EURASIP Journal on Applied Signal Processing, vol. 2004, no. 13, pp. 2025-2033, 2004.

[34] S. Barbarossa and V. Pétrone, "Analysis of polynomial-phase signals by the integrated generalized ambiguity function," IEEE Transactions on Signal Processing, vol. 45, no. 2, pp. 316327, 1997.

[35] A. Francos and M. Porat, "Analysis and synthesis of multicomponent signals using positive time-frequency distributions," IEEE Transactions on Signal Processing, vol. 47, no. 2, pp. 493504, 1999.

[36] S. Peleg and B. Friedlander, "Multicomponent signal analysis using the polynomial-phase transform," IEEE Transactions on Aerospace and Electronic Systems, vol. 32, no. 1, pp. 378-387, 1996.

[37] B. Barkat and B. Boashash, "A high-resolution quadratic timefrequency distribution for multicomponent signals analysis," IEEE Transactions on Signal Processing, vol. 49, no. 10, pp. 2232-2239, 2001.

[38] I. Djurović and L. J. Stanković, "Modification of the ICI rule-based IF estimator for high noise environments," IEEE Transactions on Signal Processing, vol. 52, no. 9, pp. 2655-2661, 2004.

[39] D. Ruppert, "Empirical-bias bandwidths for local polynomial nonparametric regression and density estimation," Journal of the American Statistical Association, vol. 92, no. 439, pp. 10491062, 1997.

[40] V. Katkovnik, "A new method for varying adaptive bandwidth selection," IEEE Transactions on Signal Processing, vol. 47, no. 9, pp. 2567-2571, 1999. 
[41] R. Kohavi, "A study of cross-validation and bootstrap for accuracy estimation and model selection," in Proceedings of the 14th International Joint Conference on Artificial Intelligence, vol. 2, pp. 1137-1143, Morgan Kaufmann Publishers, San Francisco, Calif, USA, 1995.

[42] J. Lerga, M. Vrankic, and V. Sucic, "A signal denoising method based on the improved ICI rule," IEEE Signal Processing Letters, vol. 15, pp. 601-604, 2008.

[43] I. Djurović and L. Stanković, "Influence of high noise on the instantaneous frequency estimation using quadratic timefrequency distributions," IEEE Signal Processing Letters, vol. 7, no. 11, pp. 317-319, 2000. 